\title{
Invasive DNA elements modify the nuclear architecture of their insertion site by KNOT- linked silencing in Arabidopsis thaliana
}

\author{
Stefan Grob* (D) and Ueli Grossniklaus*
}

\begin{abstract}
Background: The three-dimensional (3D) organization of chromosomes is linked to epigenetic regulation and transcriptional activity. However, only few functional features of 3D chromatin architecture have been described to date. The KNOT is a 3D chromatin structure in Arabidopsis, comprising 10 interacting genomic regions termed KNOT ENGAGED ELEMENTS (KEES). KEEs are enriched in transposable elements and associated small RNAs, suggesting a function in transposon biology.

Results: Here, we report the KNOT's involvement in regulating invasive DNA elements. Transgenes can specifically interact with the KNOT, leading to perturbations of 3D nuclear organization, which correlates with the transgene's expression: high KNOT interaction frequencies are associated with transgene silencing. KNOT-linked silencing (KLS) cannot readily be connected to canonical silencing mechanisms, such as RNA-directed DNA methylation and posttranscriptional gene silencing, as both cytosine methylation and small RNA abundance do not correlate with KLS. Furthermore, KLS exhibits paramutation-like behavior, as silenced transgenes can lead to the silencing of active transgenes in trans.

Conclusion: Transgene silencing can be connected to a specific feature of Arabidopsis 3D nuclear organization, namely the KNOT. KLS likely acts either independent of or prior to canonical silencing mechanisms, such that its characterization not only contributes to our understanding of chromosome folding but also provides valuable insights into how genomes are defended against invasive DNA elements.
\end{abstract}

Keywords: 3D nuclear organization, Arabidopsis, Gene silencing, Paramutation, Transgene, KNOT

\section{Background}

Genome organization encompasses the linear genome, the epigenome, and its three-dimensional architecture (3D genome). In contrast to the first two organizational levels, our understanding of the functional roles of the $3 \mathrm{D}$ genome is rather poor. Chromosome conformation capture (3C) technologies [1] have facilitated its exploration, implicating it in transcriptional regulation [2], replication [3], and senescence [4]. We previously proposed a role of the 3D genome in transposon biology in Arabidopsis [5]: Ten KNOT ENGAGED ELEMENTs (KEEs) (aka IHIs [6]), transposable element (TE) insertion hotspots enriched in associated small RNAs (sRNAs), contact each other to form a nuclear

\footnotetext{
* Correspondence: sgrob@botinst.uzh.ch; grossnik@botinst.uzh.ch Department of Plant and Microbial Biology \& Zurich-Basel Plant Science Center, University of Zurich, Zollikerstrasse 107, 8008 Zurich, Switzerland
}

structure termed the KNOT (Fig. 1a and Additional file 1: Table S1). The KNOT is conserved in plants, found in both dicots and monocots, and a potentially analogous structure may be formed by Drosophila piRNA clusters [5, 7].

Invasive DNA elements, such as TEs, retroviruses, and transgenes, not only are central to biotechnology but also play an important role in disease [8] and genome evolution [9]. Plants have evolved a balanced response to these elements, allowing for potential benefits, such as rapid adaptation to environmental challenges through controlled mobility [10]. In contrast, their uncontrolled proliferation and expression, which can lead to genome instability and potentially harmful ectopic gene expression, respectively, is counteracted by the silencing of invasive elements. With transgenes, silencing has been observed since the beginning of their use (reviewed in Kooter et al., [11]) and is of concern to both, gene

(c) The Author(s). 2019 Open Access This article is distributed under the terms of the Creative Commons Attribution 4.0 International License (http://creativecommons.org/licenses/by/4.0/), which permits unrestricted use, distribution, and 

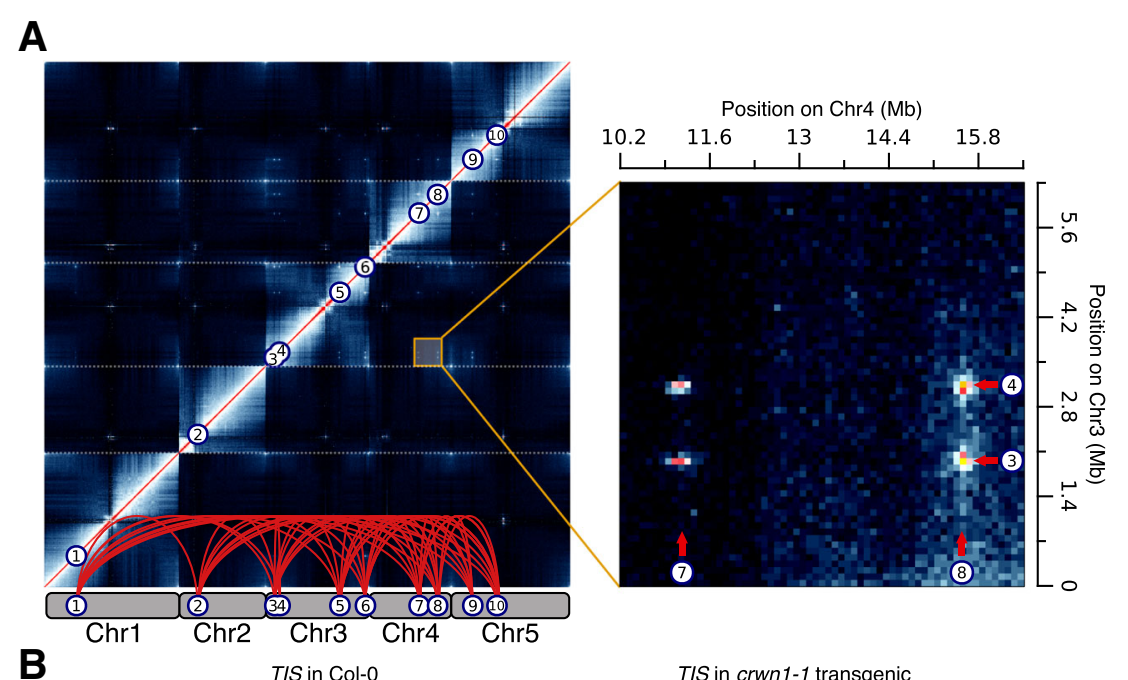

B
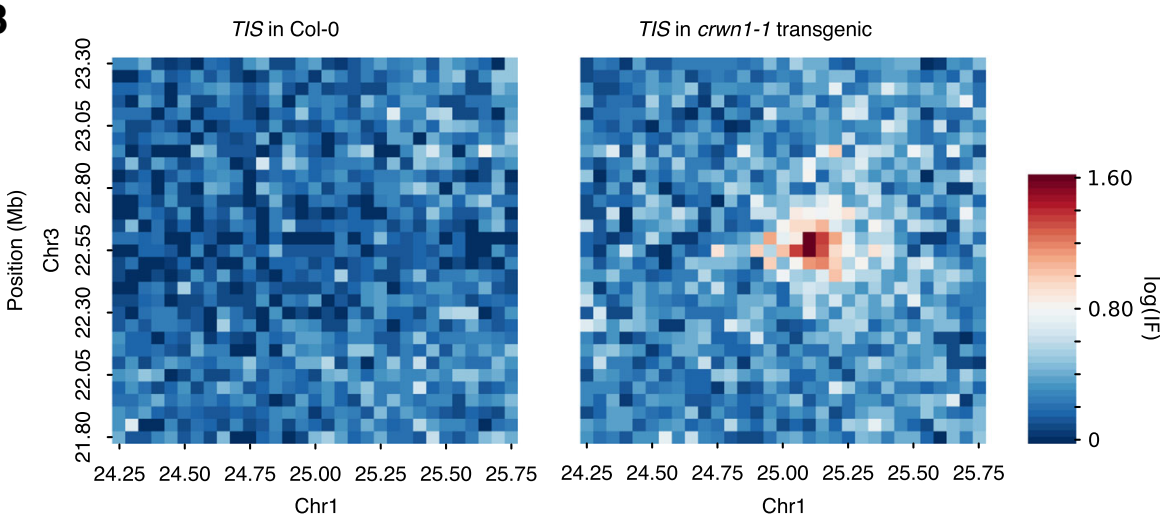

C

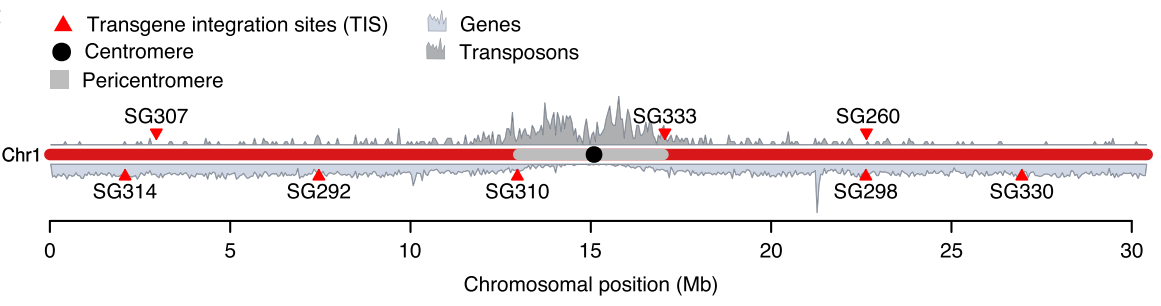

Fig. 1 Novel KNOT interactions in transgenic plants. a Left: Hi-C interactome of Arabidopsis thaliana. The KNOT is represented by network of longrange cis- and trans-contacts found between all Arabidopsis chromosomes (see also Additional file 1: Table S1). b Hi-C interaction data representing interaction frequencies (IFs) between genomic regions on chromosome 1 (Chr1) (TIS Crwn1-1, Chr1: 25151270-25156323) and Chr3 (KEE6, Chr3: 22560488-22580488). Ectopic 3D contacts can be observed between the TIS and KEE6. Left: Col-0 wild-type, Right: crwn 1-1 T-DNA insertion mutant. Interaction frequencies are pooled into 50-kb genomic bins (see also Additional file 1: Figure S1A-B). c Representation of TISs on Chr1 investigated in this study. Gene and transposon density are shown to facilitate the distinction of euchromatic and heterochromatic regions

technology and fundamental research. In plants, many transgenic approaches are based on T-DNA vectors [12]. However, despite their common origin, vectors used to generate transgenic plants exhibit significant differences with respect to transgene expression. Certain vectors, especially those containing viral $35 \mathrm{~S}$ regulatory sequences [13], such as $p R O K 2$ used to generate the insertion lines of the SALK collection [14], become more frequently silenced than others. It is unlikely that the underlying mechanism is directly associated with these transgenes, as plants must have evolved strategies to counteract invasive elements well before plant transformation was developed. Hence, although the susceptibility to silencing differs among vectors, the underlying mechanisms are likely universal, irrespective of the variation with respect to silencing. The high frequency and variability of silencing among SALK lines make them an ideal system to study the control of invasive genetic elements. Suppression of such elements in plants has been associated with sRNA-mediated processes, leading to either transcript decay or DNA methylation and transcriptional silencing $[13,15]$. Here, we introduce an alternative 
silencing phenomenon, KNOT-linked silencing (KLS), and show how transgenes and the 3D genome can reciprocally influence each other.

\section{Results \\ Ectopic 3D contacts between TRANSGENE INTEGRATION SITEs and the KNOT}

We reanalyzed previously published $\mathrm{Hi}-\mathrm{C}$ data [5] obtained from mutant plants and observed novel highfrequency long-range interactions that were absent in the wild type (Fig. 1b). In the crwn1-1 mutant [16], caused by a T-DNA insertion, these novel interactions occur between the CRWN1 locus and several KEEs. Additionally, we observed an enrichment of interaction frequencies between the TRANSGENE INTEGRATON SITE (TIS) and constitutive heterochromatin of all five Arabidopsis chromosomes (Fig. 1b and Additional file 1: Figure S1A-B).

We hypothesized that transgene integration can induce ectopic KEEs that originate from the TIS, resulting in novel high-frequency contacts between the TIS and the KNOT. Thus, transgene integration may disturb the endogenous 3D organization of the TIS. To test this hypothesis, we performed $4 \mathrm{C}$ experiments in 8 independent, publicly available transgenic SALK lines, setting the viewpoint at the respective TIS (Fig. 1c). In parallel, we generated $4 \mathrm{C}$ interaction profiles of the same viewpoints in Columbia-0 (Col-0) wild-type plants and statistically evaluated differences between transgenic and wild-type $4 \mathrm{C}$ profiles (Fig. 2). Between transgenic and wild-type lines, differential interaction analysis revealed significant differences (FDR $<0.05$ ), predominantly coinciding with KEES (6 of 8 transgenic lines) (Fig. 2). However, the magnitude of perturbation in the $4 \mathrm{C}$ profile differed considerably among lines. Three of them (SG260, SG292, and SG298) exhibited a significant change in interaction frequencies only with respect to one individual KEE (KEE3 for SG292 and KEE6 for SG260 and SG298, respectively). Other transgenic lines (SG307, SG314, and SG330) showed more severe perturbations of their 4C profile. We detected ectopic high-frequency contacts with most KEEs and with pericentromeric regions of all chromosomes, reminiscent of the initial observation in crwn1-1 (Fig. 2 and Additional file 1: Figure S1A-B). Thus, transgene integration does not solely result in the insertion of additional genetic material but can also perturb the 3D organization of the TIS in a specific manner. The absence of increased TIS-pericentromere interactions observed in SG260, SG292, and SG298 indicates that novel TIS-KEE interactions are not a consequence of TIS dislocation towards the pericentromere.

To assess whether the ectopic KEE6-TIS interactions coincide with decreased interaction frequencies between KEE6 and other KEEs, we analyzed KEE6-KNOT and KEE6-CRWN1 interaction frequencies in crwn1-1 Hi-C data and other Hi-C data sets (wild-type and transgenic) [5, 17], which did not exhibit ectopic KEE6-CRWN1 interactions. Indeed, KEE6-KNOT interaction frequencies were decreased in crwn1-1, suggesting that interactions of KEE6 with the KNOT are diluted when it also contacts the transgene (Additional file 1: Figure S1E-F).

\section{Number of insertions may influence the strength of TIS- KNOT interactions}

To further investigate variation in the extent of 3D genome perturbations between lines, we analyzed the number of TIS by Southern blotting and droplet digital PCR (ddPCR) (Additional file 1: Table S2 and Figure S2A). Transgenic lines exhibiting either no significant changes in interaction frequencies, or significant alterations with respect to single KEEs only, harbored single insertions (SG260, SG292, SG298, SG310, and SG333). All lines that exhibited more severe alterations in the 3D organization (SG307, SG314, and SG330) carried multiple insertions. PCR-based analysis using primers flanking the insertion sites indicated that multiple copies were inserted at a single locus. However, although not observed by Southern blotting, ddPCR, and short read sequencing data (4C data), we cannot completely exclude that additional T-DNA fragments are inserted elsewhere in the genome. The occurrence of large-scale rearrangements, such as translocations, can be ruled out as we can readily detect such rearrangements by $4 \mathrm{C}$ (Additional file 1: Figure S2B-D). As half of the single-insertion and all multiple-insertion lines showed high-frequency interactions with $K E E s$, transgene copy number may influence the strength but not the potential of TIS-KNOT interactions per se.

\section{TIS-KNOT 3D interactions coincide with transgene silencing}

Next, we investigated whether ectopic TIS-KEE contacts affect the activity of the transgenes. The vector $p R O K 2$, used to generate the transgenic lines [14], harbors the NPTII kanamycin resistance gene. Thus, we visually assessed the viability of transgenic seedlings grown on medium containing kanamycin (Fig. $3 \mathrm{a}$ and Additional file 1: Figure S3A). The phenotypes were uniform in three distinct populations per transgenic genotype, stressing the robustness of the transcriptional state of the transgenes (Additional file 1: Figure S3A). Viability significantly anti-correlated with TIS-KEE interactions and was strongly reduced in lines with the highest KNOT interactions, phenocopying the absence of NPTII in the wild type (Fig. 3a, b). Lines with significantly increased interaction frequencies with the KNOT but not the pericentromeres, as well as lines without increased KNOT interaction frequencies, were not significantly affected by kanamycin, thus showing sufficient NPTII 


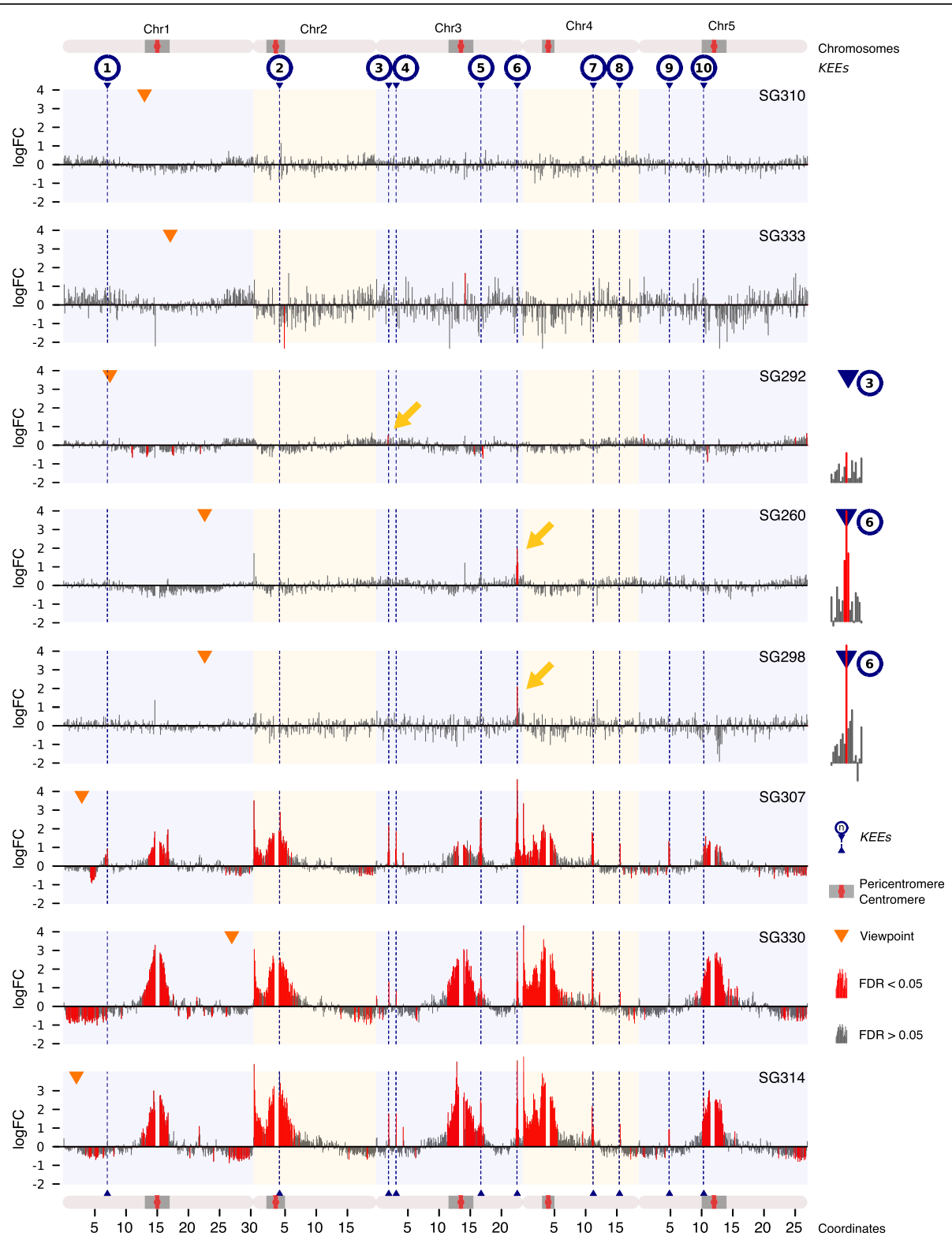

Fig. 2 TISS interact with KEES and pericentromeric regions. Differential analysis of $4 \mathrm{C}$ interactomes, including 3 wild-type and 3 transgenic $4 \mathrm{C}$ samples. Log2 fold changes (FC) are plotted. Gray: non-significant FC (FDR > 0.05). Red: significant FC (FDR $\leq 0.05)$. Orange triangles indicate viewpoints (adjacent to TIS on endogenous sequence). Blue triangles and dashed blue lines indicate positions of KEEs. Gray rectangles delineate pericentromeric regions. Interaction frequencies of single HindllI restriction fragments were pooled into 100-kb genomic bins. Yellow arrows indicate significant TIS-KEE contacts, for which magnification is given on the left

expression (Fig. 3a). We confirmed these results by RNA sequencing data, which revealed a significant anticorrelation between TIS-KEE interaction frequencies and NPTII expression (Fig. 3c) that itself significantly correlated with viability on kanamycin (Fig. 3d). As the strength of TIS-KNOT interactions negatively correlated with NPTII expression, we propose an involvement of the KNOT in transgene silencing.

Interestingly, repressive genomic neighborhoods of the TISs did not appear to affect either transgene expression or associated perturbations in TIS 3D organization: transgenes inserted into constitutive heterochromatin (SG310 and SG333) (Fig. 1c) were neither silenced nor exhibited strong 3D perturbations, whereas certain TISs in euchromatin showed significant perturbations and were silenced. To corroborate this observation, we grew 99 homozygous SALK lines carrying insertions distributed along chromosome 1 on selective medium, and scored their viability associated with NPTII expression. We did not observe decreased viability of lines that carry 


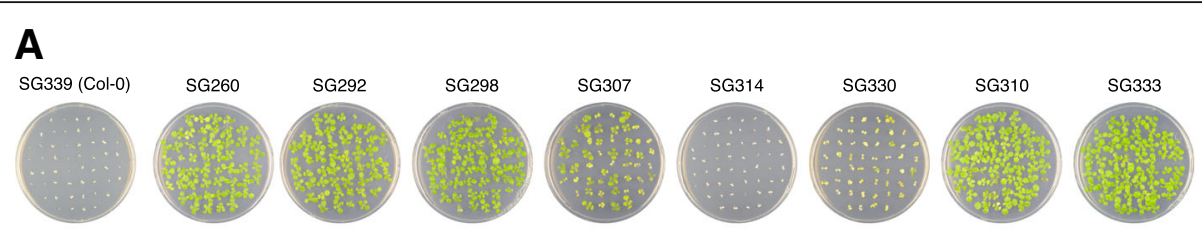

B

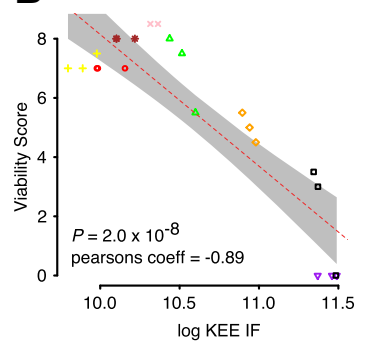

E

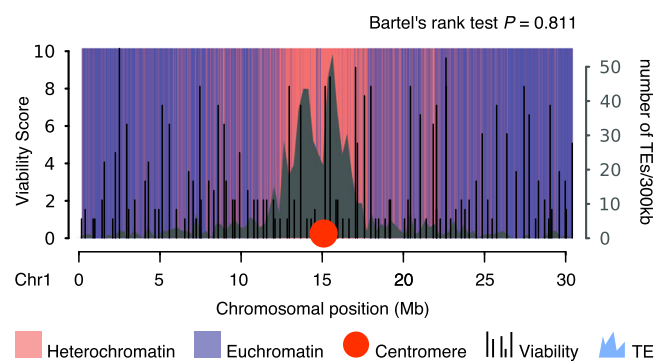

c

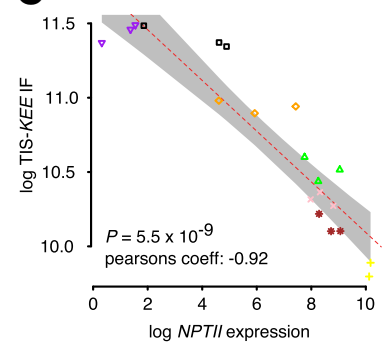

$\log$ NPTII expression

F

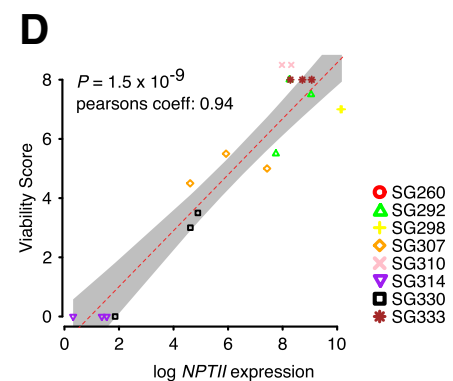

$\mathbf{F}$

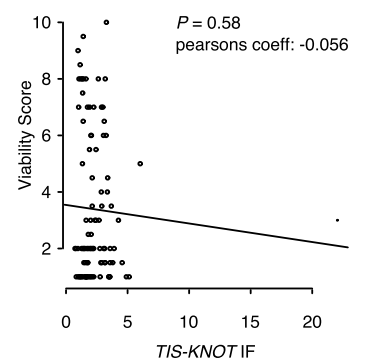

Fig. 3 Transgene silencing by KNOT-linked silencing. a Seedlings growing on medium containing kanamycin show variable resistance (see also Additional file 1: Figure S3A). b Pearson's correlation between phenotypic viability assessed by visual inspection (10—fully viable, 0—dead) in the presence of kanamycin and TIS interaction frequencies (IF) with KEES and pericentromeres (see also Additional file 1: Table S3). c Pearson's correlation between NPTII transgene expression and TIS interaction frequencies with KEEs and pericentromeres. $\mathbf{d}$ Pearson's correlation between NPTII transgene expression and phenotypically assessed kanamycin resistance (see b). e Viability score (10 - fully viable, 0 - dead) of transgenic seedling populations $(n=30)$ grown on selective medium. Transgenic lines were selected by randomly choosing a homozygous SALK line (http//signal.salkedu/cgi-bin/homozygotes.cgi) for each 300-kb genomic bin on Chr1. Numbers of transposons are indicated as a proxy for the presence of heterochromatin. Euchromatic and heterochromatic regions (purple and light red) correspond to chromatin states 1-7 and chromatin states 8-9, respectively, as previously defined [18] (see also Additional file 1: Table S4).f Pearson's correlation analysis of interaction frequencies between the prospective TIS and the KNOT in the wild type and the viability score of transgenic lines with insertions at the respective TIS. TIS-KNOT interaction frequencies were calculated from Col-0 wild-type Hi-C matrices (100 kb bins) [5]

transgenes in repressive heterochromatin (Fig. 3e). Moreover, statistical analysis rejected a non-random distribution of viability scores, a finding supported by a previous study [19]. We cannot exclude that upon transformation, chromosomal localization may have influenced transgene expression, leading to counterselection of T-DNAs inserted into repressive environments. However, as they would not have been retrieved otherwise, all transgenes analyzed here were initially expressed in $\mathrm{T}_{1}$ seedlings and acquired a distinct expression state only after their initial selection. Hence, our results suggest that at least de novo silencing of transgenes is independent of the epigenetic environment of the TIS.

Furthermore, transgene silencing cannot be predicted based on wild-type interaction frequencies of a prospective TIS and the KNOT. Using $\mathrm{Hi}-\mathrm{C}$ data from wild-type plants [5], we did not observe a significant correlation between the interaction frequencies of the prospective
TIS with the KNOT and transgene silencing (Fig. 3f), indicating that the $3 \mathrm{D}$ organization of the prospective TIS does not predispose for silencing.

To investigate whether perturbing the $3 \mathrm{D}$ organization of the TIS is limited to transgene expression or whether TIS-KNOT interactions also affect neighboring endogenous gene expression, we performed RNA sequencing. We analyzed triplicate mRNA from seven lines to test whether expression of genes surrounding the TIS differed between wild-type and transgenic lines, indicative of an effect of novel TIS-KNOT interactions. We found that transcriptional silencing is restricted to the transgene, as there was no enrichment of differentially expressed genes in the neighborhood of the TIS or the KEES (Fig. 4a). Similarly, close inspection of the transcriptional activity of neighboring genes did not reveal a significant difference between wild-type and transgenic plant lines (Fig. 4b-d). 
Endogenous loci evade KLS, indicating specificity to invasive genetic elements. Furthermore, although the genomic region encompassing the TIS and nearby genes is folded into a repressive environment, silencing is limited to the transgene itself. Thus, a perturbation of nuclear architecture alone is not sufficient to silence gene expression and other, yet to be discovered, factors may play a role in KLS specificity.

\section{Transgene silencing does not require DNA methylation}

Next, we aimed at putting KLS into the context of established silencing mechanisms in plants. There have been numerous previous reports on transgene silencing, and the underlying mechanisms have been deciphered [13]. Two principle mechanisms are proposed to initiate and/ or maintain transgene silencing: transcriptional gene silencing (TGS) and post-transcriptional gene silencing (PTGS) lead to transcriptional arrest and mRNA degradation, respectively. Homology-dependent gene silencing, another term often used for transgene silencing, can depend on either TGS [20] or PTGS [21]. It can lead to simultaneous silencing of various homologous sequences and, hence, exhibits trans-silencing effects [22, 23]. SALK T-DNA lines were found to be subject to TGS, involving the accumulation of promoter-specific sRNAs and elevated levels of cytosine methylation in transgene promoters, mediated by the RNA-directed DNA methylation (RdDM) pathway [23].

We first investigated cytosine methylation levels in the nopaline synthase promoter (nosP) driving NPTII in three active (A-lines) and three silenced lines (S-lines) by Sanger sequencing after bisulfite conversion (Fig. 5a). In average, S-lines showed elevated cytosine methylation levels and weak correlations with both, kanamycin sensitivity and KEE interaction frequencies (Fig. $5 \mathrm{a}-\mathrm{c}$ and Additional file 1: Figure S3B-E). However, SG314, exhibiting significantly higher cytosine methylation levels than all other lines, had a major effect on the statistical

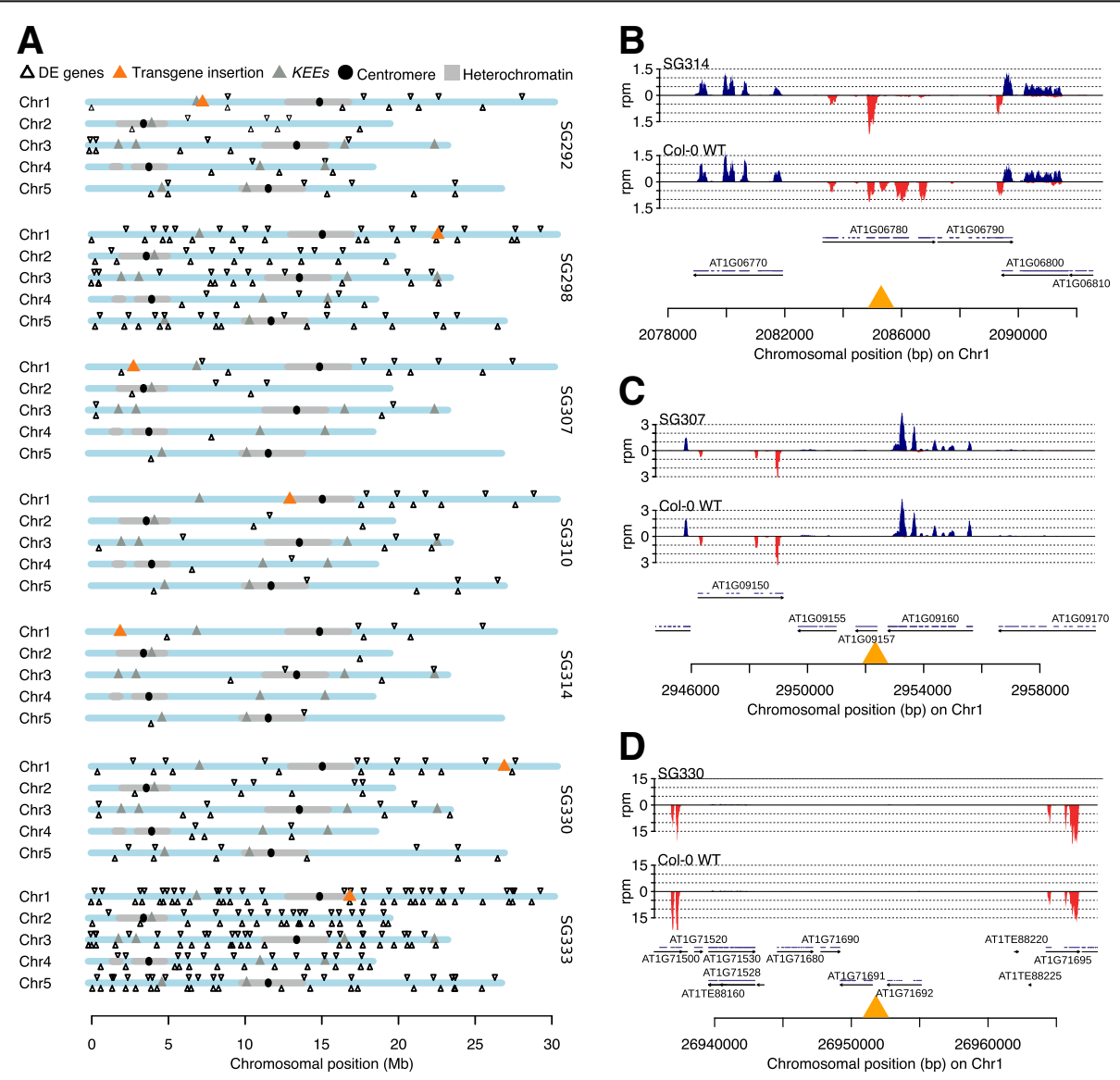

Fig. 4 Expression profiling. a Differentially expressed genes between Col-0 wild-type and transgenic lines. For each line, RNA sequencing was performed in triplicate. $\mathbf{b}-\mathbf{d}$ RNA sequencing coverage surrounding the transgene integration site. Top: transgenic line (pool of biological triplicates), bottom: Col-0 wild-type (pool of biological triplicates). Orange triangle depicts the transgene insertion site. Red and blue peaks represent (strand-specific) normalized read coverage (rpm, reads per million). Arrows mark genomic features. Blue lines represent exons. Read coverage was normalized to the total reads of triplicate RNA sequencing experiments. $\mathbf{b}$ Fourteen-kilobase region surrounding the TIS in line SG314. c Fourteen- kilobase region surrounding the TIS in line SG307. d Thirty-kilobase region surrounding the TIS in line SG330 


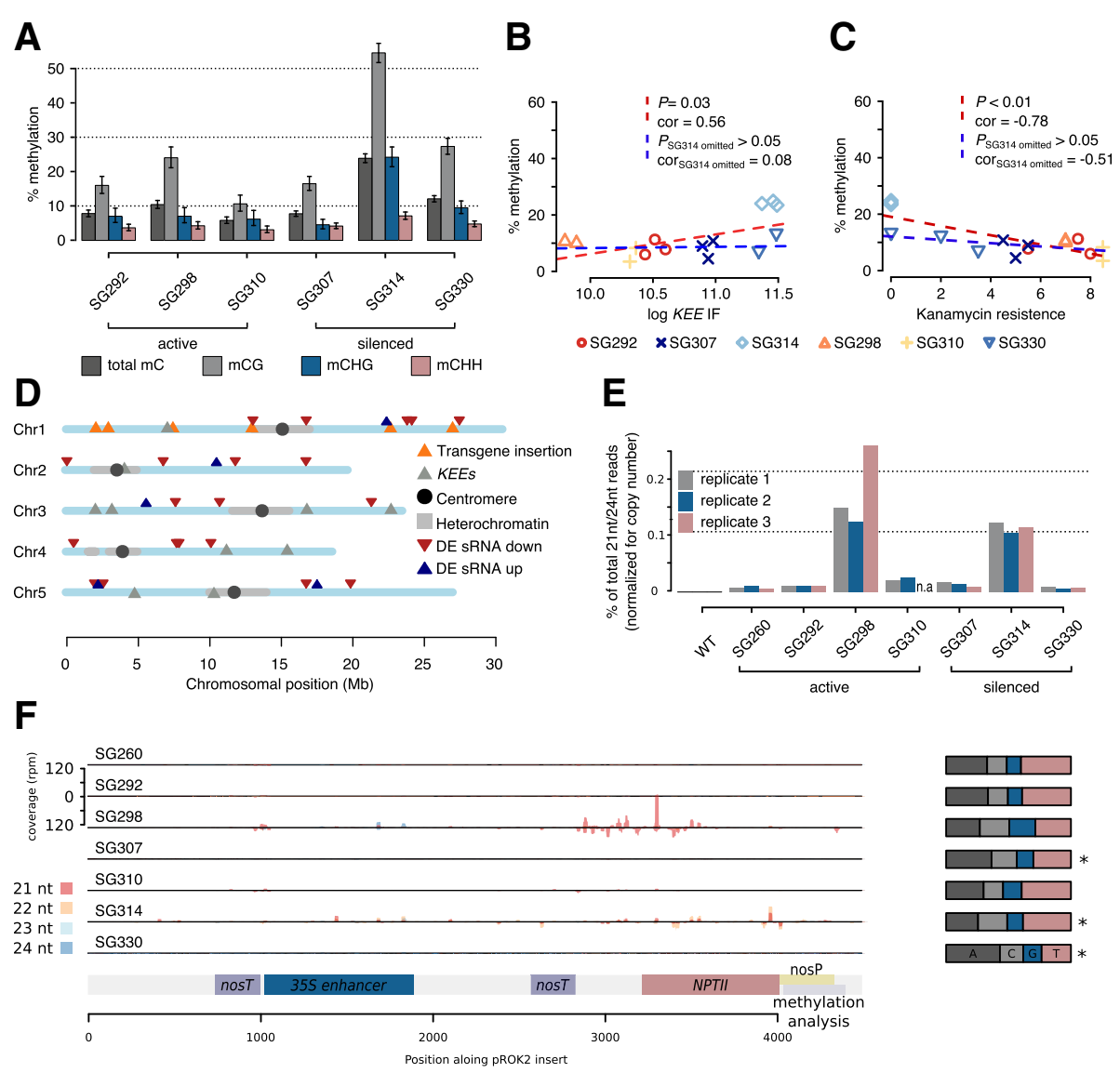

Fig. $5 \mathrm{KLS}$ is independent of canonical silencing pathways. a Bisulfite Sanger sequencing of nos $P$ (301 bp on 3 '-end of the insert). Methylation levels in all contexts significantly differed between active and silenced lines and also between individual lines (Additional file1: Table S5-6, Figure S3B-E). Error bars: Wilson 95\% confidence intervals. b Pearson's correlation analysis between 4C interaction frequencies with KEEs and pericentromeres (KEE-IF) and nosP methylation levels. Weak correlation was observed (red line). Non-significant correlation was observed when the highest methylated line (SG314) was omitted (blue line). c Correlation between kanamycin resistance phenotype and nosP methylation levels. Weak correlation was observed (red line). Non-significant correlation was observed when SG314 was omitted (blue line). d Differential analysis of sRNA-seq data. Genome-wide genomic bins (500 bp) exhibiting significant changes (logFC > 2; FDR < 0.01) between S- and A-lines (see also Additional file 1: Figure S4A). e Percentage of $21 \mathrm{nt}$ and $24 \mathrm{nt}$ sRNA-seq reads found within $p R O K 2$. For each genotype, biological triplicates were assessed (the number of reads were normalized by transgene copy number) (Additional file 1: Table S2). f Coverage of sRNA sequencing reads in 10 bp bins (21 nt- $24 \mathrm{nt}$ ) mapping to the vector pROK2. Read numbers were normalized for $P R O K 2$ copy number. The reads of the three biological replicates were pooled. Right: Summary of first nucleotides of the reads. Asterisks mark significant deviations from equal distribution of nucleotides (chi-square test, $P<0.05$ )

analysis. By omitting SG314, no significant cytosine methylation enrichment in S-lines and no significant correlation between either transgene silencing or KEE interaction frequencies and methylation levels were observed (Fig. $5 \mathrm{a}-\mathrm{c}$ ). In all transgenic lines, including SG314, the overall nosP cytosine methylation levels were lower than expected for RdDM and comparable or below average genomic methylation levels [24-27]. In summary, although one transgenic line (SG314) exhibited elevated cytosine methylation levels, which may be associated with RdDM, other silenced lines (SG307 and SG330) showed low methylation levels, indistinguishable from those of active transgenes. Therefore, although not individually tested for each investigated transgene, we conclude that cytosine methylation is not generally required for the silencing of the investigated transgenes and that methylation-dependent TGS, such as RdDM, is not a prerequisite for KLS. Consistent with these results, methylation-independent transcriptional gene silencing has previously been reported [28].

\section{sRNA abundance does not correlate with KLS}

To assess a possible involvement of sRNAs in KLS, we conducted sRNA sequencing. First, we analyzed the abundance of sRNA mapping to the $p R O K 2$ transgene. In case of a significant involvement of sRNAs in silencing the investigated transgenes, and thus KLS, we expected to find high levels of associated sRNAs in S-lines and low levels in A-lines. We detected sRNAs associated with $p R O K 2$ in all transgenic lines, although to variable 
extents (Fig. 5e, f). In accordance with our cytosine methylation analysis, sRNAs were abundant in SG314, yet no general correlation between sRNA levels and transgene silencing was found. By normalization of sRNA reads to transgene copy number, an A-line (SG298) even exhibits the highest abundance of sRNAs in all lines analyzed (Fig. 5e, f). Additionally, both the silenced lines SG330 and SG307 showed indistinguishable sRNA levels from A-lines (SG260, SG292, SG310). We conclude that sRNAs are neither sufficient nor necessary to silence these transgenes. In summary, our findings suggest that neither DNA methylation nor sRNAs play a primary role in silencing the investigated transgenes and that KLS does not depend on RdDM-related TGS.

To perform a genome-wide analysis of sRNA abundance in the investigated lines, sRNA reads were binned to 500 bp genomic regions and subsequently analyzed to detect loci of differential sRNA association (Additional file 1: Figure S4A). The sRNA profiles of active and silenced transgenic lines were very similar, identifying only few distinct differential loci (Fig. 5d). An analysis of genomic features overlapping the identified differential loci did not reveal obvious candidate factors involved in transgene silencing. We subsequently compared the identified differential sRNA loci with differentially expressed genes obtained from the mRNA-seq experiment using the same contrast (active $v s$. silenced transgenic lines), and no overlap between the two data sets was found. Similarly, analysis of the differentially expressed genes of this contrast did not provide candidates associated with transgene silencing. Our results suggest that sRNAs do not appear to be directly involved in silencing the investigated transgenes.

By performing an alternative experiment, we aimed to independently confirm that sRNAs are not a prerequisite of KLS. Specifically, we used a genetic approach to test whether PTGS is involved in KLS. As PTGS involves sRNAs that lead to mRNA decay, it can silence genes in trans. Thus, the progeny of a cross between an S- and an A-line should be at least partially silenced, as transgenes identical in sequence are present in both parental lines, such that mRNA from both transgenes should be affected by the same sRNAs. We performed reciprocal crosses using seven parental lines: one wild-type, three S-lines (SG307, SG314, SG330), and three A-lines (SG292, SG298, SG310) (Fig. 6a). This resulted in 8 progeny groups, either derived from two S-lines (SS), two A-lines (AA), two groups of progenies with parents of converse transcriptional state (SA and AS), and 4 groups of hemizygous transgenic progeny. We assessed their viability reflecting NPTII expression by growing $\mathrm{F}_{1}$ seedlings on medium containing kanamycin and by measuring the area and mean green fraction intensity of imaging data (Fig. 6a, b). The transgene expression state behaved as a heritable dominant trait (Fig. 6a). SS progeny, lacking NPTII expression, exhibited significantly reduced viability compared to all other groups, whereas SA, AS, and AA groups did not significantly differ from each other (Fig. 6b). Thus, in $F_{1}$ seedlings, KLS behaves as a recessive trait with Mendelian inheritance. This excludes the involvement of diffusible sRNAs acting in trans, suggesting that PTGS is unlikely involved in KLS.

\section{KLS shows paramutation-like features}

To assess whether the $F_{2}$ generation also follows Mendelian segregation, we cultivated progeny of the abovedescribed crosses on non-selective medium and allowed four plants of each $F_{1}$ population to self-fertilize. We then analyzed the segregation in response to kanamycin in the $\mathrm{F}_{2}$ seedling populations. Assuming Mendelian segregation, double hemizygous $\mathrm{F}_{1}$ plants containing a silenced and active NPTII transgene are expected to produce 25\% kanamycin-sensitive offspring (Additional file 1: Figure S4D). Employing PCR-based genotyping, we could confirm genetic Mendelian segregation for both transgenes (Additional file 1: Table S10). However, phenotypically, we observed a deviation from Mendelian segregation in a large fraction of the $F_{2}$ populations, manifested in significantly higher proportions (up to 92\%) of kanamycinsensitive seedlings (Fig. $6 \mathrm{c}$ and Additional file 1: Figure S4B). The observed phenotypic segregation distortion indicates that a large fraction of parentally active transgenes underwent de novo silencing, a process reminiscent of paramutation [29]. In support of our observation, trans-silencing between transgenes has been observed before [22, 23]. Importantly, during the entire crossing procedure, the trans-silencing effect depends on the initial presence of a silenced transgene, as $F_{2}$ seedling populations derived from AA crosses did not exhibit trans-silencing phenotypes (Fig. 6c). However, genotyping and subsequent quantification of NPTII transcripts by ddPCR of single $\mathrm{F}_{2}$ plants revealed that the presence of the paramutagenic allele is not necessary for the paramutagenic effect in the $F_{2}$ generation, as plants derived from AS crosses, which were homozygous for the A but lacking the $\mathrm{S}$ transgene, still exhibited full NPTII silencing (Additional file 1: Figure S4C). The observed proportions of silencing also exclude a potential dosage effect of diffusible sRNAs associated with PTGS that are produced by the parentally silenced transgene (Additional file 1: Figure S4D-E). Furthermore, the variability in silencing ratios between $F_{2}$ lines (Fig. 6c) suggests that specific genotype combinations observed in the segregating $F_{2}$ populations do not directly influence the trans-silencing potential in single $F_{2}$ plants. In summary, transgenes silenced by KLS show a paramutation-like behavior, but their initial silencing is not correlated with cytosine methylation and sRNAs targeting the transgene, 

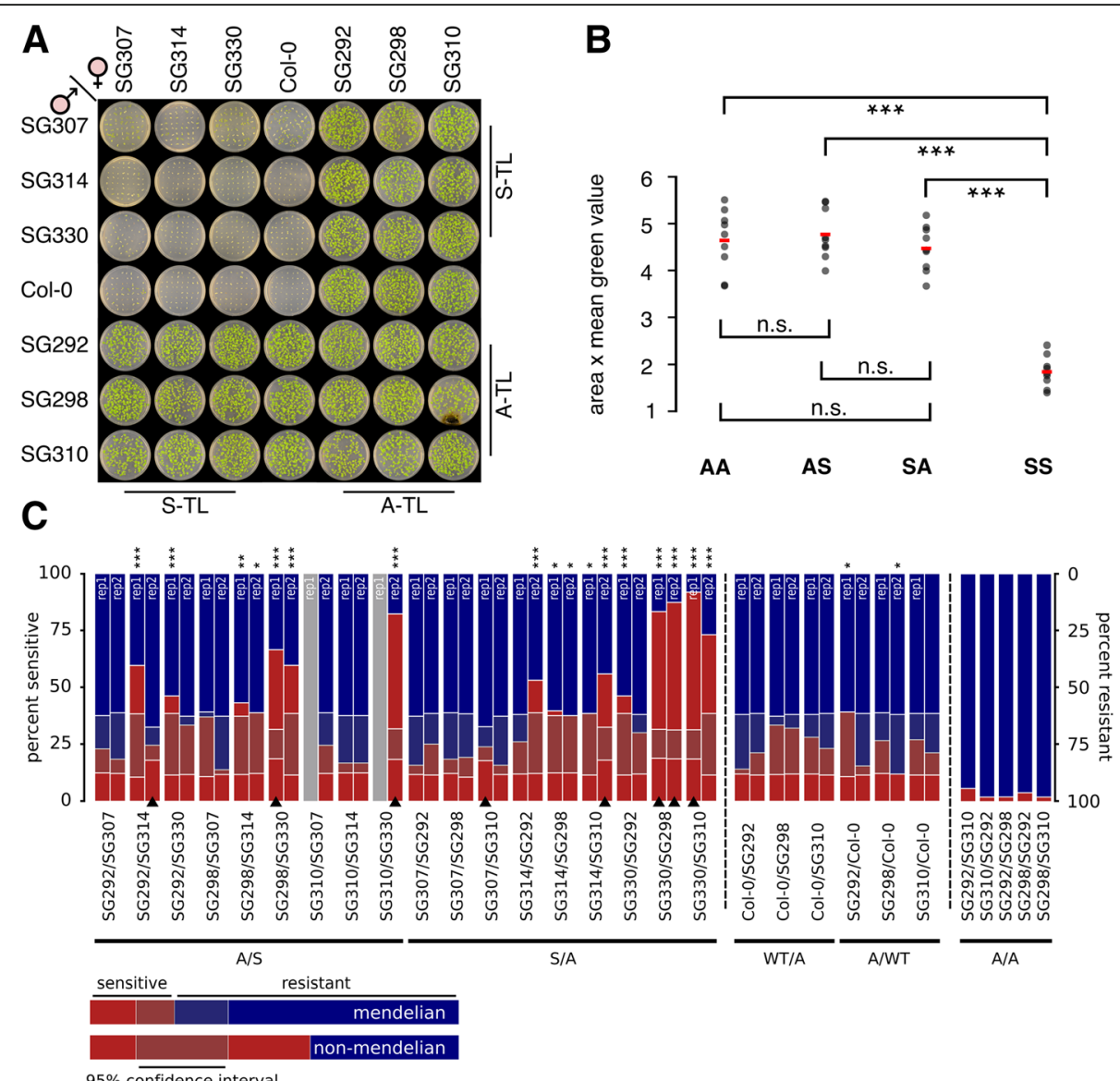

Fig. 6 Trans-silencing of transgenes. a Reciprocal crosses between silenced (S-TL) and active (A-TL) transgenic lines. Images were acquired from 14-day-old seedlings. b Area and mean "green" value were assessed by ImageJ. Student's $t$ tests were performed to assess significant differences between all quarters

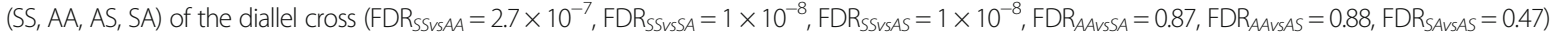
(Additional file 1: Table S7). c Segregation in $\mathrm{F}_{2}$ seedlings. Chi-square tests were performed to test for deviation from Mendelian segregation (null-hypothesis $0.25 / 0.75$ (sensitive/resistant), ${ }^{*} 0.05>P \geq 0.01,{ }^{* *} 0.01>P \geq 0.001,{ }^{* * *} P<0.001$ ). Confidence interval indicates the range in which Mendelian segregation cannot be rejected. Bars with black triangles stem from pooled data of 4 individual $F_{1}$ siblings ( $n=$ up to $4 \times 52$ seedlings), non-marked bars stem from mixed seeds of the 4 siblings ( $n=$ up to 52 seedlings). Gray bars: data not available (Additional file 1: Table S8, Table S9, Figure S4B)

indicating a novel phenomenon depending on 3D genome interactions.

\section{Discussion}

\section{The KNOT is a novel player of the genome's defense} system

Our results suggest that insertion of transgenes has more profound effects on genome structure than previously anticipated, as not only genetic material is added, but also the 3D architecture of the TIS can be severely perturbed. These alterations have a profound impact on the transgenes themselves, as architectural perturbations can clearly be associated with the expression state of the transgenes. Importantly, the observed perturbations are not random. Moreover, we detected specific ectopic interactions with the KNOT, suggesting its involvement in the nuclear defense system against invasive genetic elements.
KLS does not depend on canonical silencing pathways

In our studies on the nature of KLS, we could not find strong evidence for an involvement of either PTGS or canonical TGS, suggesting that KLS is at least initially independent of these silencing mechanisms. In support, a previous study showed that the number of KEEs is not reduced in mutants leading to the de-repression of silenced genes [6]. The epigenetic marks affected in these mutants include repressive histone modifications, such as H3K27me3 (clf;swn double mutant) and H3K9me2 (suvh4;suvh5;suvh6 triple mutant); DNA methylation (ddm1, met1, and cmt3 mutants); and epigenetic processes affecting silencing by other means (mom1 mutant). This suggests that epigenetic marks commonly associated with gene silencing, such as H3K9me2, H3K27me3, and cytosine methylation, are not necessary for interactions among KEEs. Hence, an involvement of these canonical repressive marks in the recruitment of 
T-DNAs to the KNOT, thereby initiating KLS, is unlikely. Interestingly, in many of these mutants, an identical set of ectopic KEEs can be observed in apparently pre-defined positions, which show a significant enrichment of VANDAL6 and ATLANTYS3 TEs, both of which are highly enriched in the ten canonical KEEs (Additional file 1: Figure S4F). This finding suggests that inactive KEE regions exist in the genome, whose functional activation may rely on active transcription of TEs.

\section{KLS is a dynamic process}

We observed that TIS-KNOT interactions alone are insufficient for transgene silencing, which only occurs in lines that also acquired high-frequency TIS-pericentromere interactions. We hypothesize that TIS-KNOT interactions may initiate transgene silencing, which could then be followed by a secondary alteration of the TIS 3D organization, leading to a tight association with constitutive heterochromatin and complete silencing of the transgene. Although not yet observed at the time of writing, continuous growing of lines showing exclusively TIS-KNOT interactions, such as SG298 and SG260, over several generations may corroborate this hypothesis.

KEEs vary among each other with respect to their chromosomal position and epigenetic characteristics [5]. Whereas a subset of KEEs originate from heterochromatic regions and exhibit contact frequency biases towards other heterochromatic regions, the majority of KEEs are situated in euchromatic chromosome arms, accompanied by more frequent contacts with euchromatin (Additional file 1: Figure S4G). KEE6, which plays the most prominent role in ectopic TIS-KEE contacts (Fig. 2), equally interacts with hetero- and euchromatic regions (Additional file 1: Figure S4G), despite its localization on a euchromatic chromosome arm. We speculate that KEE6 may shuttle between both hetero- and euchromatin and, hence, may represent a key KEE in KLS.

KLS shows a paramutation-like behavior, whereby the transcriptional state of one transgene can be transferred to another. The KLS trans-silencing activity differs from classical paramutation, as it affects non-homologous loci and seems to depend on passage through an additional generation, the latter having also been observed for other transgenes with paramutation-like behavior in Arabidopsis [30]. Furthermore, the maintenance of the repressed paramutated state in maize requires factors involved in the biogenesis of $24 \mathrm{nt}$ long sRNAs, which are homologous to components of the RdDM pathway in Arabidopsis [31]. Similarly, sRNAs have previously been implicated in homology-dependent trans-silencing in Arabidopsis [22, 23]. In contrast to these findings, sRNAs do not seem to play a determining role in KLS, making their involvement in KLS-related trans-silencing unlikely. Importantly, 3D chromosome folding has previously been reported to be involved in paramutation, specifically in cis-interactions between the maize $b 1$ locus affected by paramutation and its regulatory sequences [32]. Similarly, 3D architecture has been found to play a role in paramutation-like interactions between Polycomb group response element-containing (trans) genes in Drosophila [33].

Our work may stimulate future studies elucidating the regulatory mechanisms of paramutation-like phenomena in plants, especially concerning the developmental timing of its establishment and maintenance. The observation that not all $F_{2}$ offspring derived from a single $F_{1}$ plant exhibit de novo silencing of the transgene suggests that KLS may be established after floral induction. In contrast, decisions on its maintenance may occur in the vegetative shoot meristem because all $F_{3}$ offspring from a single $F_{2}$ plant show a uniform phenotype. However, silencing is not inherited in all $\mathrm{F}_{3}$ populations derived from $F_{2}$ plants with two silenced $A$ alleles as some pedigrees showed a reversion to the active state (Additional file 1: Figure $\mathrm{S} 4 \mathrm{H}$ ). It is worth noting that reversion was only observed in the pedigree of SG310xSG330 crosses but not SG298xSG330 crosses. In contrast to SG310, which showed no significant alteration of the TIS's 3D profile, SG298 exhibited increased TIS-KNOT interactions (Fig. 2), which might facilitate the stable silencing of the transgene.

Transgene silencing represents an acquired epigenetic state, which is stably inherited over subsequent generations. All the transgenic lines analyzed here initially exhibited active NPTII transcription [14]; hence, KLS is a dynamic process, potentially established and augmented over consecutive generations. Previous reports on transgenerational epigenetic inheritance implicated DNA methylation in this process $[34,35]$. Our results suggest an independent role for 3D genome organization in the transgenerational epigenetic inheritance of silenced transgenes. Although we observed tight TIS-KEE interactions being stably inherited over subsequent generations, we also show that KLS may contribute to the plasticity of transgenerational epigenetic inheritance through a paramutation-like trans-silencing mechanism and its reversion.

\section{Molecular mechanism underlying KLS remains to be deciphered}

Very likely, KLS involves a set of protein cofactors that mediate 3D TIS-KEE interactions. The identification of these cofactors will be essential for a better understanding of KLS and its embedding within other nuclear processes. However, this search will be challenging due to the technical inaccessibility of KLS phenotypes, such as TIS-KEE interactions, for large-scale genetic screening. 
KLS represents a previously uncharacterized phenomenon to defend the genome against invasive DNA elements. Hence, KLS not only is important for a basic understanding of gene regulation in the context of the $3 \mathrm{D}$ genome but also is of great interest to plant biotechnology, as transgene integration may have a larger impact on genome architecture than previously thought.

\section{Conclusions}

Mobile invasive DNA elements can threaten proper genome function. Hence, their transcription is regulated and can be shut down by cellular processes known as gene silencing mechanisms. We here present a novel aspect of gene silencing, which is linked to the KNOT, a specific 3D chromosomal structure. Our results suggest a functional role of $3 \mathrm{D}$ genome folding in the defense against invasive DNA elements. KLS appears to be independent of previously published silencing mechanism, whose hallmarks are increased DNA methylation and RNA interference. In fact, KLS may even underlie these silencing mechanisms and represent an initial stage in recognizing foreign DNA elements. Interestingly, the KNOT is conserved within the plant kingdom, such that KLS may represent a basal silencing mechanism common to most plants.

\section{Methods}

\section{Plant material}

Seeds of transgenic Arabidodpsis thaliana lines were acquired through the European Arabidopsis Stock Center (NASC) (http://arabidopsis.info/) (Additional file 1: Table S16). All parental lines were genotyped, and homozygous individuals were selected and selfed to produce $F_{1}$ seeds. Plants were grown under long-day conditions $\left(16 \mathrm{~h}\right.$ light, $8 \mathrm{~h}$ dark, $22^{\circ} \mathrm{C}$ day, $18^{\circ} \mathrm{C}$ night). Seeds were sterilized using hypochloric acid and stratified for 2 days at $4{ }^{\circ} \mathrm{C}$. All plant material used in this study stems from 14-day-old seedlings cultivated as previously described [36]. All analyzed Arabidopsis lines are in the Columbia-0 (Col-0) accession.

\section{$4 C$ experiments}

Generation of $4 \mathrm{C}$ templates was performed as previously described [36]. To minimize technical biases, we placed the $4 \mathrm{C}$ viewpoints adjacent to the TIS on endogenous DNA sequence; thus, the $4 \mathrm{C}$ template enrichment could be performed using identical primer pairs in transgenic and wild-type $4 \mathrm{C}$ samples. All $4 \mathrm{C}$ experiments were performed in biological triplicates. Primer sequences and restriction enzymes used in the $4 \mathrm{C}$ experiments are indicated in Additional file 1: Table S12. 4C sequencing reads were aligned using bowtie [37] with the parameters -a $-\mathrm{v} \quad 0-\mathrm{m} 25$ (no mismatches allowed, multiple alignments allowed). Alignment scores, plant lineage, and $4 \mathrm{C}$ replicate information are summarized in Additional file 1: Table S17. Reads with multiple alignments were weighted using Rcount-multireads [38]. Weighted reads were mapped to individual restriction fragments using HiCdat [39], yielding tables describing the number of reads, and thus interaction frequencies (IFs) per individual HindIII restriction fragment. The IFs were subsequently allocated to non-overlapping 100-kb genomic bins. IFs were further processed using edgeR [40] to determine count data (log count per million) and to perform differential analysis between transgenic and wildtype $4 \mathrm{C}$ interaction profiles, comprising of 3 biological replicates each. For this, count data was normalized for library sizes (edgeR: calcNormFactors()), followed by estimating common and tag-wise dispersion (edgeR: estimateCommonDisp() and estimateTagwiseDisp()). Differential analysis was then performed using the exact test (edgeR: exactTest()). Significant differences in IFs between transgenic and wild-type data sets were defined by a false discovery rate (FDR) $<0.05$ (using BenjaminiHochberg-adjusted $P$ values).

\section{$\mathrm{Hi}-\mathrm{C}$ data and virtual $4 \mathrm{C}$ analysis}

Previously published $\mathrm{Hi}-\mathrm{C}$ interaction data [5] were processed as previously described [5]. Hi-C snapshots were taken from regions of interest using a 50-kb (Fig. 1b) and 100-kb (Fig. 1a) binning size. Virtual 3C and $4 \mathrm{C}$ analysis (Additional file 1: Figure S1A and Fig. 3f) was performed by extracting the genomic $100-\mathrm{kb}$ bin relevant to the viewpoint of interest (crwn1-1: Chr1 25.1-25.2 Mb (Additional file 1: Figure S1A) or summing up Hi-C interaction frequencies between TIS and bins $(100 \mathrm{~kb})$ encompassing KEEs and pericentromeres (Fig. 3f; KEE1 and the pericentromere of chromosome 1 were omitted). To determine KEE6-KNOT contact frequencies, IFs were extracted from previously published $\mathrm{Hi}-\mathrm{C}$ matrices at 50-kb resolution [5, 17], without using distance normalization. KEE6-KNOT IFs were defined as contact frequencies between KEE6 and KEE1, KEE2, KEE3, KEE4, KEE5, KEE7, KEE8, KEE9, and KEE10. Contact frequencies between KEEs and hetero- and euchromatic regions (defined in [5]), respectively, were extracted from $\mathrm{Hi}-\mathrm{C}$ data of $50-\mathrm{kb}$ bin size and are shown in Additional file 1: Figure S4G. Contact frequencies stemming from cis-contacts and contacts between KEEs were excluded from this analysis.

\section{Copy number analysis}

The copy numbers of inserted transgenes were assessed using Southern blot analysis and droplet digital PCR. DNA for both types of analysis was extracted from 14day-old Arabidopsis seedlings using a MasterPure DNA purification kit (Epicentre, Madison, WIS, USA). 


\section{Southern blot}

For Southern blot analysis, genomic DNA was digested using the HindIII restriction enzyme (New England Biolabs, Ipswich, MA, USA). The digestion efficiency was analyzed on $1.5 \%$ agarose gel. Subsequently, the gel was washed for $10 \mathrm{~min}$ in $0.25 \mathrm{M} \mathrm{HCl}$, followed by 15 min incubation in denaturation solution $(1.5 \mathrm{M} \mathrm{NaCl}$, $0.5 \mathrm{~N} \mathrm{NaOH}$ ) and 15-min incubation in neutralization solution (1.5 M NaCl, $1 \mathrm{M}$ TrisHCl, $\mathrm{pH} 7.5)$. The fragmented and denatured DNA was then transferred to a positively charged nylon membrane (Roche, Basel, Switzerland) overnight at room temperature (RT). After rinsing the nylon membrane in $2 \times$ SSC buffer, the DNA was UV crosslinked (GS cross linker BioRad (BioRad, Hercules, CA, USA)). The membrane was then placed in a glass cylinder and incubated in $15 \mathrm{ml}$ of hybridization solution (DIG Easy Hyb Granules, Roche, Basel, Switzerland) for $5 \mathrm{~h}$ at $42^{\circ} \mathrm{C}$ under constant rotation. Following pre-hybridization, the membrane was incubated in $15 \mathrm{ml}$ of fresh hybridization solution containing $8 \mu \mathrm{l}$ of Salmonsperm-DNA and $5 \mu \mathrm{l}$ of digoxigenin (DIG)-labeled probe (generated by incorporation of DIG-labeled dUTP (Roche, Basel, Switzerland)) at $42{ }^{\circ} \mathrm{C}$ overnight. The probe was generated using the following primer pair: forward primer: GTCAAGAAGGCGATAGAAGGCG, reverse primer: GCTTGGGTGGAGAG GCTATT and covers large parts of the NPTII gene. The next day, the membrane remaining in the glass cylinder was washed two times with W1 $(2 \times$ SSC, $0.1 \%$ SDS $)$ for $5 \mathrm{~min}$ at $68{ }^{\circ} \mathrm{C}$, followed by 15 -min washing in W2 $(0.2 \times$ SSC, $0.1 \% \mathrm{SDS})$ at $68^{\circ} \mathrm{C}$, and $15 \mathrm{~min}$ in W3 $(0.1 \times \mathrm{SSC}$, $0.1 \%$ SDS). Subsequently, the membrane was transferred to a plastic tray and incubated at RT in WB (100 mM maleic acid, $150 \mathrm{mM} \mathrm{NaCl}, 0.3 \%$ Tween-20, pH 7.5), followed by $30 \mathrm{~min}$ in B2 $(2 \mathrm{~g}$ Roche Blocking Reagent (Roche, Basel, Switzerland) in $200 \mathrm{ml} \mathrm{B1} \mathrm{(100} \mathrm{mM}$ maleic acid, $150 \mathrm{mM} \mathrm{NaCl}, \mathrm{pH} 7.5)$ ). Then, $1.5 \mu \mathrm{l}$ antiDIG-alkaline phosphatase conjugate (Roche, Basel, Switzerland) in $50 \mathrm{ml} \mathrm{B2}$ was added and the membrane was incubated for $30 \mathrm{~min}$. The membrane was washed three times for $40 \mathrm{~min}$ in WB and then incubated for 5 min in B3 $(100 \mathrm{mM}$ TrisHCl, $100 \mathrm{mM} \mathrm{NaCl}, 50 \mathrm{mM}$ $\mathrm{MgCl}_{2}, \mathrm{pH}$ 9.5). Finally, the membrane was overlaid with $6 \mathrm{ml}$ of substrate solution $(60 \mu \mathrm{l}$ CDP Star (Roche, Basel, Switzerland) in $6 \mathrm{ml} \mathrm{B3}$ ) and subsequently exposed in a trans-illuminator (Biorad Chemidoc XRS, (BioRad, Hercules, CA, USA)). Image acquisition was conducted after $10,000 \mathrm{~s}$ of exposure.

\section{Droplet digital PCR}

Droplet digital PCR (ddPCR) was performed to quantify NPTII (and thus transgene) copy number using a Biorad QX200 Droplet Digital PCR system (BioRad, Hercules, CA, USA). The concentration of NPTII (transgene), FIE
(AT3G20740; endogenous single copy gene), and LYS (AT5G62150; endogenous single copy gene) was assessed using $2 \mathrm{ng}$ of input genomic DNA. The rounded average between NPTII/FIE and NPTII/LYS ratios was finally used to determine the NPTII copy number. Droplet generation was performed according to the manufacturer's protocol. Following droplet generation, the templates were amplified in a T100 thermal cycler (BioRad, Hercules, CA, USA). Fluorescence reads of the individual droplets were analyzed using Quanta Soft v1.7 (BioRad, Hercules, CA, USA). For each sample and probe, experiments were performed in technical duplicates. Primer and probe sequence information is shown in Additional file 1: Table S14. Probes were custom designed and acquired from Life Technologies (Life Technologies, ThermoFisher Scientific, Waltham, MA, USA).

\section{mRNA sequencing}

RNA was extracted from 14-day-old Arabidopsis seedlings using RNeasy Plant Mini Kit (Qiagen, Venlo, The Netherlands). RNA was extracted from three $F_{1}$ seedling populations per following parental plant lines: SG339 (wild type), SG292, SG298, SG307, SG310, SG314, SG330, and SG333. After library preparation using the Illumina Stranded mRNA RNA-seq protocol, total RNA was subjected to Illumina RNA sequencing (RNA-seq). RNA-seq reads were aligned using the subjunc [41] RNA sequencing read alignment program. The numbers of valid alignments are shown in Additional file 1: Table S18. Aligned RNA-seq reads were then weighted and mapped to individual transcriptional units (genes, TEs) using Rcount [38]. The preprocessed transcription data was analyzed using the edgeR [40] differential expression (DE) analysis program. DE was analyzed for two types of contrasts: (i) individual parental transgenic lines (using three $F_{1}$ seedling populations) $v s$. Col-0 wild type, and (ii) all combined transgenic lines $v s$. wild type. To analyze DE, we chose a standard approach using general linearized models. After estimating common and trended (edgeR: estimateGLMCommonDisp() and estimateGLMTrendedDisp()) dispersion, we applied a gene-wise negative binomial generalized linear model (edgeR: glmfit(), followed by $\operatorname{glmLRT}())$ to assess DE. $P$ values were adjusted according to BenjaminiHochberg, and DE genes exhibiting adjusted $P$ values < 0.05 were scored as significant.

\section{sRNA-seq analysis}

Total RNA was extracted from 14-day-old Arabidopsis seedlings using the mirVana miRNA isolation kit (Ambion, ThermoFisher Scientific, Waltham, MA, USA). RNA was extracted from three $F_{1}$ seedling populations of the following parental lines: SG339 (Col-0 wild type; SG339A, SG339B, SG339C), SG260 (SG261, SG368, 
SG371), SG292 (SG335, SG337, SG369), SG298 (SG350, SG361, SG362), SG307 (SG342, SG355, SG356), SG310 (SG310, SG340), SG314 (SG346, SG355, SG356), and SG330 (SG352, SG353. SG359).

Total RNA was ligated to Illumina sequencing adapters, size selected, and subsequently sequenced on Illumina HighSeq 2500. The adapters of Illumina sequencing reads were trimmed using cutadapt (parameters: $-\mathrm{m} 17$-q 20; adapter sequence: TGGAATTCTCGGGTGCCAAGGAACTCCAGTCAC). Subsequently, the trimmed reads were filtered by aligning them against regions encompassing rRNA genes (10 kb surrounding them, Chr2,1..10000, Chr3,14194000..14204000) and tRNA genes, as well as chloroplast and mitochondrial sequences. The unaligned reads were size selected (17-30 bp) using an awk command and subsequently aligned to the Arabidopsis reference genome (TAIR10) using bowtie with the following parameters: bowtie $-\mathrm{v} 2$-best $-\mathrm{m}$ 10,000 (allowing two mismatches and up to 10,000 equally best alignments). The aligned sequencing reads were corrected for multiple alignment using Rcount-multireads and subsequently binned into 500-bp non-overlapping genomic bins (see also Additional file 1: Table S19). Differential analysis was performed using edgeR with the same parameters as described for differential RNA-seq analyses. To find genomic features associated with differential 500-bp bins, bedtools [42] intersect has been employed.

\section{NPTII expression by ddPCR}

Total RNA was extracted using the standard Trizol RNA extraction protocol, followed by RNA purification using a Direct-zol Micro Prep kit (Zymo Research, CA, USA). To remove residual DNA, RNA samples were treated with $2 \mathrm{U}$ of TURBO DNase following the manufacturer's protocol (Invitrogen, ThermoFisher Scientific, Waltham, MA, USA). Ten microliters (ca. $1 \mu \mathrm{g}$ ) of purified RNA samples were incubated for $10 \mathrm{~min}$ at $70^{\circ} \mathrm{C}$ with $1 \mu \mathrm{l}$ oligodT, $1 \mu \mathrm{l}$ RNase OUT (ThermoFisher Scientific, Waltham, MA, USA). Reverse transcription was performed using SuperScriptII reverse transcriptase, following the manufacturer's protocol (ThermoFisher Scientific, Waltham, MA, USA). ddPCR was performed amplifying both NPTII transcripts and $U B C 9$ transcripts as internal control to normalize for different amount of input material.

\section{Methylation analysis}

DNA from 14-day-old Arabidopsis seedlings was extracted using a MasterPure DNA extraction kit (Epicentre, Madison, WIS, USA). The DNA was bisulfite converted using an EpiTect Bisulfite Kit (Qiagen, Venlo, Netherlands) according to the manufacturer's protocol. Bisulfiteconverted DNA was amplified using a Kapa Library Amplification Kit (Kapa Biosystems, Wilmington, MA, USA).
Primer sequences are indicated in Additional file 1: Table S15. PCR products (see also Fig. 5f) were subsequently purified from an agarose gel, cloned into the pJet1.2 cloning vector (CloneJET PCR cloning Kit, ThermoFisher Scientific, Waltham, MA, USA), and transformed into DH5 $\alpha$ E. coli cells. Subsequently, the extracted vectors were subjected to Sanger sequencing. The resulting sequences were trimmed and preprocessed using BISMA (http://services.ibc.uni-stuttgart.de/BDPC/BISMA/) [43]. CG, CHG, and CHH methylation levels were assessed using Kismeth (http://katahdin. mssm.edu/kismeth/revpage.pl) [44]. Statistical analysis of the methylation data was performed as previously described [45, 46]. Methylation data of all $F_{1}$ samples belonging to the same parental line were pooled, and subsequently Wilson's 95\% confidence interval was calculated using the $\mathrm{R}$ package "binom." Chloroplast DNA does not exhibit cytosine methylation; thus, a region of chloroplast DNA was amplified, cloned, and sequenced to assess the bisulfite conversion efficiency (see also Additional file 1: Table S5 and S6).

\section{Kanamycin sensitivity phenotype analysis Visual assessment}

Kanamycin sensitivity in parental lines was analyzed by visual inspection. An experimenter unaware of the experimental design was asked to judge general viability of the seedlings using previously acquired images and rate the viability between 0 (dead) and 10 (perfectly viable) (double-blind assay).

\section{Image data analysis}

Images were acquired from 14-day-old seedlings grown on kanamycin-containing medium. The images were further processed and analyzed using the Image image analysis software. The color images were split in red, green, and blue channels. All subsequent steps were conducted in the green channel images. To extract the area covered by seedling tissue, a gray threshold was set, which has been previously empirically defined and showed the best separation between seedling tissue and background (lower threshold 160, upper threshold 255). The total area and the mean gray value within the threshold were measured. The product between total area and mean gray value (area $\times$ mean; Additional file 1 : Table S7) was used to perform statistical analysis. Assuming normal distribution of the data, we performed cross-wise $t$ tests between progeny classes (progeny classes derived from crosses with the wild type were omitted). The $P$ values were adjusted for multiple testing using the Benjamini-Hochberg (aka FDR) algorithm. Statistical testing was conducted using $\mathrm{R}$.

\section{Statistical analysis}

Correlation between viability on kanamycin (as well as NPTII expression) and TIS-KNOT IFs was performed 
using the R-base cor.test() function. The slope and intercept were retrieved by employing a linear model using the R-base $\operatorname{lm}()$ function. To assess whether the chromosomal position may affect transgene expression, the viability score of 99 transgenic lines inserted into chromosome 1 were assessed visually. Subsequently, chromosome ordered viability scores were tested for randomness using a two-sided Bartels rank test.

\section{Additional file}

Additional file 1: Supplementary tables (Tables S1-S19) and figures (Figures S1-S4). (PDF $5719 \mathrm{~kb}$ )

\section{Abbreviations}

3D: Three-dimensional; 4C: Circular chromosome conformation capture; Aline: Active transgenic line; ddPCR: Droplet digital PCR; FC: Fold change; IF: Interaction frequency; KEEs: KNOT ENGAGED ELEMENTS; KLS: KNOT-linked silencing; nosP: Nopaline synthase promotor; PTGS: Post-transcriptional gene silencing; RdDM: RNA-dependent DNA methylation; S-line: Silenced transgenic line; sRNA: Small RNA; sRNA-seq: sRNA sequencing; TE: Transposable element; TGS: Transcriptional gene silencing; TIS: TRANSGENE INTEGRATION SITE

\section{Acknowledgements}

We thank F. Fiscalini for the help with genotyping; V. Gagliardini for setting up the ddPCR assays; A. Hermann for the help with crosses; A. Sarazin for the advice on sRNA-seq; C. Eichenberger, A. Bolaños, D. Guthörl, A. Frey, and P. Kopf for the general laboratory support; $\mathrm{P}$. Jullien for the viability scoring; and P. Jullien, J. Vermeer, Ö. Kartal, H. Vogler, and M. Schmid for valuable input on the manuscript.

\section{Authors' contributions}

SG and UG conceived the study. SG designed and performed the experiments, analyzed the data, and wrote the manuscript. UG acquired funding and helped with data interpretation and writing of the manuscript. All authors read and approved the final manuscript.

\section{Funding}

This work was supported by the University of Zurich and an Advanced Grant of the European Research Council (MEDEA-250358) to U.G.

\section{Availability of data and materials}

4C and RNA, and sRNA sequencing data are publicly available at the Sequence Read Archive (SRA; https://www.ncbi.nlm.nih.gov/sra/) under accession SRP126992 [47]. Codes for data processing and analysis are available on Zenodo [48].

\section{Ethics approval and consent to participate}

Not applicable

\section{Consent for publication}

Not applicable

\section{Competing interests}

The authors declare that they have no competing interests.

Received: 30 November 2018 Accepted: 22 May 2019

Published online: 11 June 2019

\section{References}

1. Schmitt AD, Hu M, Ren B. Genome-wide mapping and analysis of chromosome architecture. Nat Rev Mol Cell Biol. 2016;17:743-55.

2. Gorkin DU, Leung D, Ren B. The 3D genome in transcriptional regulation and pluripotency. Stem Cell. 2014;14:762-75.
3. Pope BD, Ryba T, Dileep V, Yue F, Wu W, Denas O, et al. Topologically associating domains are stable units of replication-timing regulation. Nature. 2014;515:402-5.

4. Pombo A, Dillon N. Three-dimensional genome architecture: players and mechanisms. Nat Rev Mol Cell Biol. 2015;16:245-57.

5. Grob S, Schmid MW, Grossniklaus U. Hi-C analysis in Arabidopsis identifies the KNOT, a structure with similarities to the flamenco locus of Drosophila. Mol Cell. 2014;55:678-93.

6. Feng S, Cokus SJ, Schubert V, Zhai J, Pellegrini M, Jacobsen SE. Genomewide $\mathrm{Hi}-\mathrm{C}$ analyses in wild-type and mutants reveal high-resolution chromatin interactions in Arabidopsis. Mol Cell. 2014;55:694-707.

7. Dong Q, Li N, Li X, Yuan Z, Xie D, Wang X, et al. Genome-wide Hi-C analysis reveals extensive hierarchical chromatin interactions in rice. Plant J. 2018:94:1141-56.

8. Hancks DC, Kazazian HH. Roles for retrotransposon insertions in human disease. Mob DNA. 2016;7:1-28.

9. Feschotte C, Pritham EJ. DNA transposons and the evolution of eukaryotic genomes. Annu Rev Genet. 2007:41:331-68.

10. Ito H, Kakutani T. Control of transposable elements in Arabidopsis thaliana. Chromosom Res. 2014;22:217-23.

11. Kooter JM, Matzke MA, Meyer P. Listening to the silent genes: transgene silencing, gene regulation and pathogen control. Trends Plant Sci. 1999:4:340-7.

12. Zupan JR, Zambryski P. Transfer of T-DNA from Agrobacterium to the plant cell. Plant Physiol. 1995;107:1041-7.

13. Rajeevkumar $S$, Anunanthini P, Sathishkumar R. Epigenetic silencing in transgenic plants. Front Plant Sci. 2015:6:524-8.

14. Alonso JM, Stepanova AN, Leisse TJ, Kim CJ, Chen H, Shinn P, et al. Genome-wide insertional mutagenesis of Arabidopsis thaliana. Science. 2003;301:653-7.

15. Borges F, Martienssen RA. The expanding world of small RNAs in plants. Nat Rev Mol Cell Biol. 2015;16:727-41.

16. Dittmer TA, Stacey NJ, Sugimoto-Shirasu K, Richards EJ. LITTLE NUCLEI genes affecting nuclear morphology in Arabidopsis thaliana. Plant Cell. 2007;19: 2793-803.

17. Moissiard G, Cokus SJ, Cary J, Feng S, Billi AC, Stroud H, et al. MORC family ATPases required for heterochromatin condensation and gene silencing. Science. 2012:336:1448-51.

18. Sequeira-Mendes J, Aragüez I, Peiró R, Mendez-Giraldez R, Zhang X, Jacobsen SE, et al. The functional topography of the Arabidopsis genome is organized in a reduced number of linear motifs of chromatin states. Plant Cell. 2014:26:2351-66.

19. Schubert D, Lechtenberg B, Forsbach A, Gils M, Bahadur S, Schmidt R. Silencing in Arabidopsis T-DNA transformants: the predominant role of a gene-specific RNA sensing mechanism versus position effects. Plant Cell. 2004; 16:2561-72.

20. Matzke MA, Primig M, Trnovsky J, Matzke AJM. Reversible methylation and inactivation of marker genes in sequentially transformed tobacco plants. EMBO J. 1989:8:643-9.

21. Jauvion V, Rivard M, Bouteiller N, Elmayan T, Vaucheret H. RDR2 partially antagonizes the production of RDR6-dependent siRNA in sense transgenemediated PTGS. PLoS One. 2012;7:7-11

22. Daxinger $L$, Hunter $B$, Sheikh $M$, Jauvion V, Gasciolli V, Vaucheret $H$, et al. Unexpected silencing effects from T-DNA tags in Arabidopsis. Trends Plant Sci. 2008;13:4-6.

23. Mlotshwa S, Pruss GJ, Gao Z, Mgutshini NL, Li J, Chen X, et al. Transcriptional silencing induced by Arabidopsis T-DNA mutants is associated with 355 promoter siRNAs and requires genes involved in siRNAmediated chromatin silencing. Plant J. 2010;64:699-704.

24. Kanno T, Bucher E, Daxinger L, Huettel B, Böhmdorfer G, Gregor W, et al. A structural-maintenance-of-chromosomes hinge domain-containing protein is required for RNA-directed DNA methylation. Nat Genet. 2008;40:670-5.

25. Greaves IK, Groszmann M, Wang A, Peacock WJ, Dennis ES. Inheritance of trans chromosomal methylation patterns from Arabidopsis F1 hybrids. Proc Natl Acad Sci. 2014;111:2017-22.

26. Lister R, O'Malley RC, Tonti-Filippini J, Gregory BD, Berry CC, Millar AH, et al. Highly integrated single-base resolution maps of the epigenome in Arabidopsis. Cell. 2008:133:523-36.

27. Aufsatz W, Mette MF, Matzke AJM, Matzke M. The role of MET1 in RNAdirected de novo and maintenance methylation of CG dinucleotides. Plant Mol Biol. 2004;54:793-804.

28. Diéquez MJ, Vaucheret H, Paszkowski J, Mittelsten Scheid O. Cytosine methylation at CG and CNG sites is not a prerequisite for the initiation of 
transcriptional gene silencing in plants, but it is required for its maintenance. Mol Gen Genet. 1998;259:207-15.

29. Hollick JB. Paramutation and related phenomena in diverse species. Nat Rev Genet. 2016;18:5-23.

30. Mittelsten Scheid O, Afsar K, Paszkowski J. Formation of stable epialleles and their paramutation-like interaction in tetraploid Arabidopsis thaliana. Nat Genet. 2003;34:450-4.

31. Giacopelli BJ, Hollick JB. Trans-homolog interactions facilitating paramutation in maize. Plant Physiol. 2015;168:1226-36.

32. Louwers M, Bader R, Haring M, Van Driel R, De Laat W, Stam M. Tissue- and expression level-specific chromatin looping at maize $b 1$ epialleles. Plant Cell. 2009;21:832-42.

33. Ciabrelli F, Comoglio F, Fellous S, Bonev B, Ninova M, Szabo Q, et al. Stable Polycomb-dependent transgenerational inheritance of chromatin states in Drosophila. Nat Genet. 2017:49:876-86.

34. Mirouze M, Reinders J, Bucher E, Nishimura T, Schneeberger K, Ossowski S, et al. Selective epigenetic control of retrotransposition in Arabidopsis. Nature. 2009;461:427-30

35. Heard E, Martienssen RA. Transgenerational epigenetic inheritance: myths and mechanisms. Cell. 2014;157:95-109.

36. Grob S, Schmid MW, Luedtke NW, Wicker T, Grossniklaus U. Characterization of chromosomal architecture in Arabidopsis by chromosome conformation capture. Genome Biol. 2013;14:R129.

37. Langmead B, Trapnell C, Pop M, Salzberg SL. Ultrafast and memory-efficient alignment of short DNA sequences to the human genome. Genome Biol. 2009;10:R25.

38. Schmid MW, Grossniklaus U. Rcount: simple and flexible RNA-Seq read counting. Bioinformatics. 2015;31:436-7.

39. Schmid MW, Grob S, Grossniklaus U. HiCdat: a fast and easy-to-use Hi-C data analysis tool. BMC Bioinformatics. 2015;16:277.

40. Robinson MD, McCarthy DJ, Smyth GK. edgeR: a Bioconductor package for differential expression analysis of digital gene expression data. Bioinformatics. 2010;26:139-40.

41. Liao Y, Smyth GK, Shi W. The Subread aligner: fast, accurate and scalable read mapping by seed-and-vote. Nucleic Acids Res. 2013;41:e108.

42. Quinlan AR, Hall IM. BEDTools: a flexible suite of utilities for comparing genomic features. Bioinformatics. 2010;26:841-2.

43. Rohde C, Zhang Y, Reinhardt R, Jeltsch A. BISMA--fast and accurate bisulfite sequencing data analysis of individual clones from unique and repetitive sequences. BMC Bioinformatics. 2010;11:230.

44. Gruntman E, Qi Y, Slotkin RK, Roeder T, Martienssen RA, Kismeth SR. Analyzer of plant methylation states through bisulfite sequencing. BMC Bioinformatics. 2008;9:314-71.

45. Henderson IR, Deleris A, Wong W, Zhong X, Chin HG, Horwitz GA, et al. The de novo cytosine methyltransferase DRM2 requires intact UBA domains and a catalytically mutated paralog DRM3 during RNA-directed DNA methylation in Arabidopsis thaliana. PLoS Genet. 2010;6:1001182.

46. Jullien PE, Susaki D, Yelagandula R, Higashiyama T, Berger F. DNA methylation dynamics during sexual reproduction in Arabidopsis thaliana. Curr Biol. 2012:22:1825-30.

47. Grob S, Grossniklaus U. Invasive DNA elements modify the nuclear architecture of their insertion site by KNOT-linked silencing in Arabidopsis thaliana. mRNA, 4C, and sRNA data sets, sequence read archive (SRA). 2019. https://www.ncbi.nlm.nih.gov/bioproject/PRJNA422972.

48. Grob S, Grossniklaus U. Invasive DNA elements modify the nuclear architecture of their insertion site by KNOT-Linked Silencing in Arabidopsis thaliana. Zenodo. https://doi.org/10.5281/zenodo.3147387. Accessed March 2019.

\section{Publisher's Note}

Springer Nature remains neutral with regard to jurisdictional claims in published maps and institutional affiliations.

Ready to submit your research? Choose BMC and benefit from:

- fast, convenient online submission

- thorough peer review by experienced researchers in your field

- rapid publication on acceptance

- support for research data, including large and complex data types

- gold Open Access which fosters wider collaboration and increased citations

- maximum visibility for your research: over $100 \mathrm{M}$ website views per year

At BMC, research is always in progress.

Learn more biomedcentral.com/submissions 